LA-UR-02-6807

Approved for public release; distribution is unlimited.

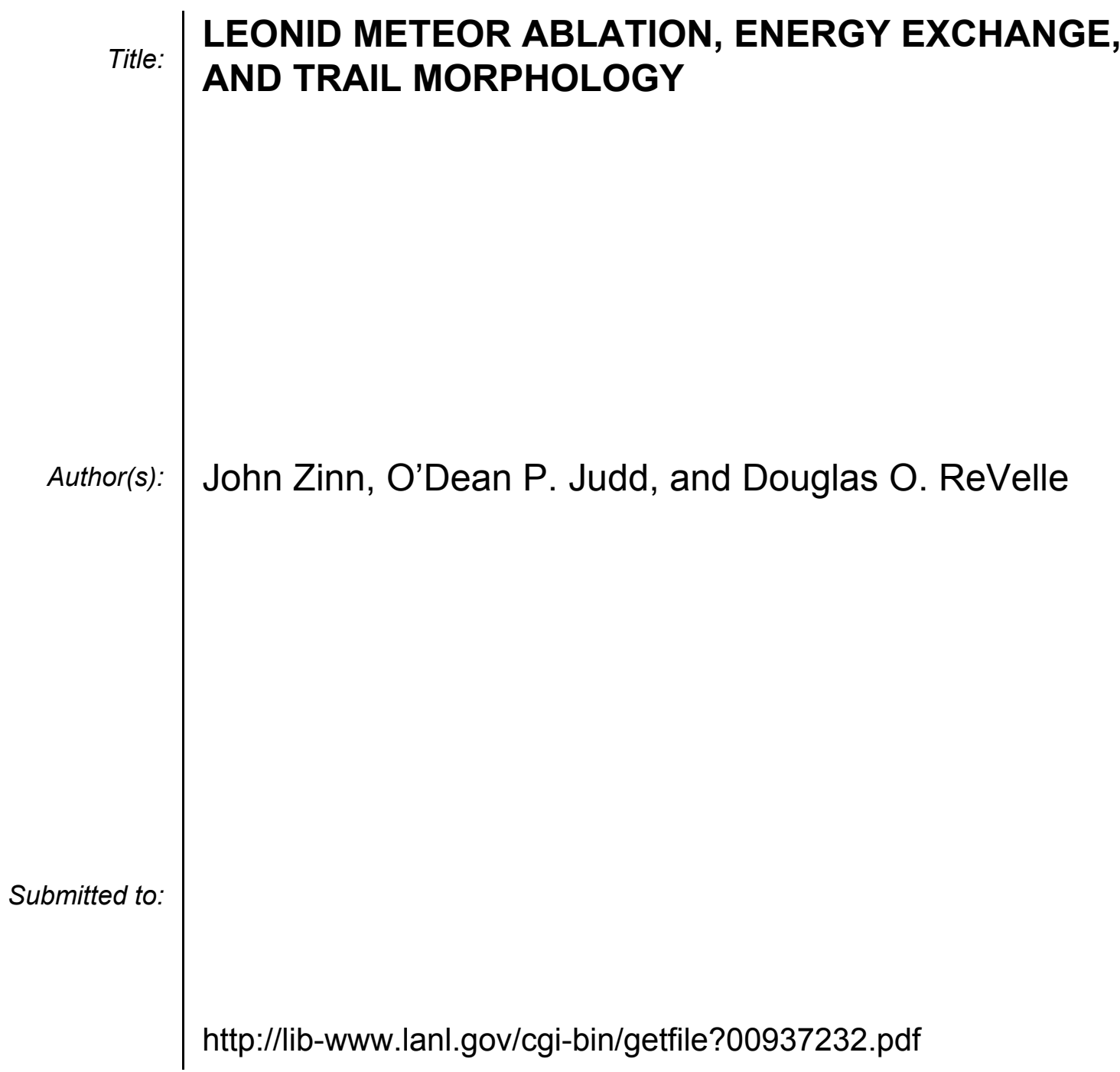

Los Alamos National Laboratory, an affirmative action/equal opportunity employer, is operated by the University of California for the U.S. Department of Energy under contract W-7405-ENG-36. By acceptance of this article, the publisher recognizes that the U.S. Government retains a nonexclusive, royaltyfree license to publish or reproduce the published form of this contribution, or to allow others to do so, for U.S. Government purposes. Los Alamos National Laboratory requests that the publisher identify this article as work performed under the auspices of the U.S. Department of Energy. Los Alamos National Laboratory strongly supports academic freedom and a researcher's right to publish; as an institution, however, the Laboratory does not endorse the viewpoint of a publication or guarantee its technical correctness. 


\title{
LEONID METEOR ABLATION, ENERGY EXCHANGE, AND TRAIL MORPHOLOGY
}

\author{
John Zinn, O’Dean P. Judd, and Douglas O. ReVelle
}

Los Alamos National Laboratory, Los Alamos, NM 87545

\begin{abstract}
This paper describes theoretical model studies of the interaction of Leonid meteoroids with the earth's atmosphere. Subject to some modest-to-strenuous approximations we compute the rates of ablation and deceleration, energy deposition, and terminal altitudes of the meteors as functions of their initial mass and bulk density, velocity, trajectory entry angle, drag coefficient, heat of ablation, and an ablation energy transfer fraction. We find that the dominant energy deposition in the atmosphere is associated with the stopping of the ablated meteor particles and vapor by the surrounding air. Then having computed the energy deposition rates versus altitude we compute the hydrodynamic and radiative expansion of the hot wake material in the radial direction, along with the associated air chemistry. From the computed results we can then plot two-dimensional temperature contours - as functions of the instantaneous distance behind the meteor and radial distance from the center of the wake, at various altitudes along the meteor's path. We also compute the rates of emission of radiation and the radiative efficiency, and discuss comparisons with observations.
\end{abstract}

\section{INTRODUCTION}

This paper describes some theoretical studies of the interaction of Leonid meteors with the earth's atmosphere — at relatively low altitudes (below $90 \mathrm{~km}$ ) where the hydrodynamic fluid approximations apply. The subject divides naturally into two parts. The first deals with the ablation/vaporization of the solid meteor material, and the rapid stopping of the ablated vapors by the atmosphere, and the conversion of the vapor kinetic energy into internal energy of the vapor and entrained air. The second part treats the approximately cylindrical expansion of the hot vapor-air mixture into the surrounding air - a process that involves radiation transport and hydrodynamics, and associated air chemistry.

\section{ABLATION AND SLOWING DOWN OF THE SOLID METEOR BODY}

It should be noted at the outset that for very high velocity bodies the energy required to completely vaporize the solid meteor is orders of magnitude smaller than the initial meteor kinetic energy. In the case of Leonids we will find that the meteor itself is slowed down hardly at all before it is completely ablated. The ablated material is born with a forward velocity equal to the velocity of the meteor, and the predominant process involved in deposition of the meteor energy in the atmosphere is the conversion of the kinetic energy of the ablating meteor particles/vapor as they interact with (i.e. are stopped by) the background air. The vapor and particles are stopped very quickly, so that they, together with the energy, remain behind in the meteor wake.

Our analysis for the motion and ablation of the solid meteor body follows closely after that of Bronshten (1983), Ceplecha et al, (1998), and references therein. But we have made some simplifications. For a meteor of instantaneous mass $m$, velocity $v$, and projected surface area $S$, moving on a trajectory with zenith angle $\theta$, through the atmosphere with local density $\rho$ with a drag coefficient $C_{D}$ the equation of motion is (see Landau and Lifshitz, 1959)

$$
m \frac{d v}{d t}=-\frac{1}{2} C_{D} \rho v^{2} S+m g \cos \theta
$$


where $g$ is the acceleration due to gravity, and we have assumed a straight linear trajectory and a flat earth. For the case of Leonids we can almost always ignore the mg term. Then the rate of loss of kinetic energy of the solid meteor, Q, corresponding to Eq. (1) is

$$
\frac{d Q}{d t}=-\frac{1}{2} C_{D} \rho v^{3} S
$$

Some of this energy goes into heating and ablation (i.e. surface spallation and vaporization) of the meteor, and some goes into the air. If a fraction $f_{Q}$ of the energy goes into ablation of the meteor, and if the heat of ablation is $e$, then the rate of loss of meteor mass by ablation is given by

$$
\frac{d m}{d t}=-\frac{f_{Q}}{2 e} C_{D} \rho v^{3} S
$$

Upon dividing Eq. (1) (without the mg term) by Eq. (3) and rearranging terms we obtain

$$
v d v=\frac{e}{f_{Q}} \frac{d m}{m}
$$

Then if $\frac{f_{Q}}{e}$ is taken to be constant we can integrate Eq. (4), with the result

$$
v^{2}-v_{\infty}^{2}=\frac{2 e}{f_{Q}} \ln \frac{m}{m_{\infty}}
$$

where $m_{\infty}$ and $v_{\infty}$ are the initial mass and velocity of the meteor before encountering the atmosphere.

In the case of Leonids, for which $v_{\infty}$ is quite large (about $70 \mathrm{~km} / \mathrm{s}$, or $7 \times 10^{6} \mathrm{~cm} / \mathrm{s}$ ), unless $\frac{f_{Q}}{e}$ is smaller than about $1 \times 10^{-12} \mathrm{~s}^{2} / \mathrm{cm}^{2}$ the velocity remains essentially constant until the meteor is almost completely ablated away.

Now returning to Eq. (3), if we take the velocity $v$ to be constant, and take the meteor trajectory to be straight and oriented with given zenith angle $\theta$, then the rate of change of altitude is

so that then from Eq. (3)

$$
\frac{d z}{d t}=-v_{\infty} \cos \theta
$$

$$
\frac{d m}{d z}=\frac{f_{Q} C_{D} \rho v_{\infty}^{2} S}{2 e \cos \theta}
$$

If we make the further approximation that the meteoroid is a sphere, and does not fragment, and remains spherical as it ablates, then the meteor mass is $m=\frac{4}{3} \pi \rho_{s} r^{3}$, and $S=\pi r^{2}$, where $r$ is the radius and $\rho_{s}$ is the bulk density of the solid meteor. It follows that

$$
S=1.209 \frac{m^{2 / 3}}{\rho_{s}^{2 / 3}}
$$

Further, assuming that the atmosphere is locally exponential,

$$
\rho=\rho_{0} \exp \left\{-\left(z-z_{0}\right) / H\right\}
$$

where $\rho_{\mathrm{o}}$ and $H$ are the local atmospheric density and scale height at a reference altitude $z_{0}$. ( $H$ is a slowly-varying function of z.) Then we can rewrite the $d m / d z$ equation as

$$
m^{-2 / 3} d m=\frac{.604 f_{Q} C_{D}}{e \rho_{s}^{2 / 3}} \frac{\rho_{0} v_{\infty}^{2}}{\cos \theta} \exp \left\{-\left(z-z_{0}\right) / H\right\} d z
$$

Now defining $A \equiv \frac{.604 f_{Q} C_{D}}{e \rho_{s}^{2 / 3}}$ and assuming $A=$ constant, Eq. (10) can be integrated to give

$$
m^{1 / 3}=m_{\infty}^{1 / 3}-\frac{H A}{3} \frac{\rho_{0} v_{\infty}^{2}}{\cos \theta} \exp \left\{-\left(z-z_{0}\right) / H\right\} .
$$


that

The bottom end of the meteor trail is where $m=0$ (altitude $z=z_{T}$ ). Setting $\mathrm{m}=0$ in the above equation we find

$$
z_{T}=z_{0}+H \ln \left(\frac{H A m_{\infty}{ }^{-1 / 3}}{3} \frac{\rho_{0} v_{\infty}^{2}}{\cos \theta}\right)
$$

It's also of interest to inquire at what altitude the rate of mass loss is a maximum. If we call that altitude $z_{m}$, we find after some algebra that

$$
z_{m}=z_{T}+H \ln 3
$$

\section{STOPPING OF THE ABLATED METEOR VAPOR}

The point of this section is to show that the ablated meteor vapor, which is born with the velocity of the meteor itself, is very quickly stopped by the background air. In the process its kinetic energy is converted into internal energy of the vapor-air mixture, which is left in the wake behind the meteor.

To show this qualitatively, consider a small element of ablated meteor vapor - e.g. the amount that is given off over one centimeter distance along the meteor track. Let its mass be called $m_{m}$ and its instantaneous velocity be $v$. As it moves it sweeps up background air, and let the instantaneous mass of that air be $m_{a}$. Then since momentum is conserved

$$
\frac{d}{d t}\left[\left(m_{a}+m_{m}\right) v\right]=0
$$

and since $m_{m}$ is constant it follows that

$$
\frac{d v}{v}=-\frac{d m_{a}}{\left(m_{a}+m_{m}\right)}
$$

which, by integration gives

$$
v=v_{o}\left(\frac{m_{m}}{m_{a}+m_{m}}\right),
$$

where $v_{o}$ is the initial velocity.

So by the time the vapor cloud element has swept up a mass of air equal to its own mass the velocity will have dropped by a factor of two. The kinetic energy will decrease accordingly. If we ignore energy losses by radiation then we would expect energy to be conserved, so that the internal energy of the cloud (vapor-air mixture) would increase by the same amount as the kinetic energy decreased. Then the specific internal energy in the system $(E$ in $\mathrm{ergs} / \mathrm{g}$ ) would be given by

$$
E=\frac{1}{2} m_{m} v_{o}^{2}\left\{\frac{m_{a}}{\left(m_{m}+m_{a}\right)^{2}}\right\}
$$

According to this expression, taking $v_{o}=70 \mathrm{~km} / \mathrm{s}$, and using a real air equation of state, by the time that $m_{a}=$ $m_{m}, E$ would be $6 \times 10^{12} \mathrm{erg} / \mathrm{g}$, and the corresponding temperature would be $3.6 \mathrm{eV}$.

As the cloud heats up it expands in the crosswise dimension. As a rough approximation, suppose that the crosswise velocity is equal to the thermal speed (which approximates the hydrodynamic expansion velocity).

$$
v_{\text {thermal }}=\sqrt{\frac{3 k T}{m_{\text {atom }}}}=C \sqrt{E},
$$

where $k$ is Boltzmann's constant and $m_{\text {atom }}$ is the average air atomic mass, and $C=\sqrt{\frac{3 k}{m_{\text {atom }} C_{p}}}$ and where $C_{p}$ is the effective specific heat. Accordingly the cross-sectional area of the cloud element $S_{c}$ will increase with time at a rate given by

$$
\frac{d S_{c}}{d t}=2 C \sqrt{\pi S_{c} E}
$$

The mass of swept-up air increases at a rate given by

$$
\frac{d m_{a}}{d t}=\rho S_{c} v,
$$

where $\rho$ is the local density of the background air, which varies with altitude as in Eq. (9). The altitude of the cloud, $z_{c}$, decreases with time as given by

$$
\frac{d z_{c}}{d t}=-v \cos \theta .
$$


So then there are three simultaneous differential equations to solve, together with some algebraic expressions. They are easily integrated with a small computer code. Without going into details, the results show that even at very high altitudes the vapor picks up air mass very quickly and slows down quickly, and the internal energy rises very fast, leading to rapid increase in the cross-sectional area $S_{c}$, which facilitates further air pickup. As an example, if a one-gram gas cloud were released at $100 \mathrm{~km}$ altitude with a velocity of $70 \mathrm{~km} / \mathrm{s}$ it would be essentially stopped by the time it reached $99.5 \mathrm{~km}$. (And, of course, in the process the vapor is left behind by the meteor.) The internal energy density in the cloud is a maximum at the point where it has picked up a mass of air equal to its own mass. At that point its velocity has dropped by a factor of two and the temperature is about $3.7 \mathrm{eV}$.

\section{ENERGY DEPOSITION ALONG THE METEOR TRAJECTORY}

The analysis in the previous section is crude, but it was intended only to demonstrate that as soon as any mass is ablated (vaporized) the moving vapor is stopped quickly by the surrounding air and its kinetic energy is converted into internal energy of the meteor-vapor-air cloud. More than $99 \%$ of the energy deposited in the air by the meteor (in the case of Leonids) is associated with the stopping of this ablated vapor. The "drag energy" transferred directly to the air by the solid meteor (Eq. (2)) is negligible by comparison.

The rate of meteor mass loss by ablation per unit length of the meteor trajectory is $-\frac{d m}{d s}$ where $d s=-\frac{d z}{\cos \theta}$, so that then the rate at which energy is released into the meteor-vapor-air cloud is given by

$$
\frac{d U}{d s}=-\frac{v_{\infty}^{2}}{2} \frac{d m}{d s}=\frac{A}{2} \rho v_{\infty}^{4}
$$

down to the terminal altitude $z_{T}$. The integral of $\frac{d U}{d s}$ from the terminal altitude $z_{T}$ up to $z=\infty$ is equal to $\frac{1}{2} m_{\infty} v_{\infty}^{2}$.

\section{EMPIRICAL EVALUATION OF THE PARAMETER $\boldsymbol{A}$ FOR LEONIDS}

Now the values of the parameters $f_{Q}, e, C_{D}$, and $\rho_{s}$ are not accurately known. However, the combined parameter $A$ should have close to the same value for all meteors of a given family (e.g. Leonids). Thus, if we can determine $A$ for one Leonid case, that value should be applicable for all Leonids. For any one Leonid, if we have measured the mass $m_{\infty}$ (e.g. from an infrasound record) and $\cos \theta$ (zenith angle of the radiant), and the terminal altitude $z_{T}$, then we can determine $A$ from Eq (12). In Eq (12), if we set the reference altitude $z_{0}$ equal to the measured terminal altitude $z_{T}$, then we obtain

$$
A=\frac{3 m_{\infty}{ }^{1 / 3}}{H} \frac{\cos \theta}{\rho_{0} v_{\infty}^{2}}
$$

where $\rho_{\mathrm{o}}$ and $H$ are the atmospheric density and scale height at the terminal altitude $z_{T}$. Here we are of course assuming that the meteor did not fragment. If it did, Eq. (23) will give a wrong result.

There are quite detailed observational data on a large Leonid seen over, New Mexico at 3:06 AM on 17 November 1998, and described partially in [Zinn et al, 1999, and Drummond et al, 2001]. For that meteor the trajectory was found from video measurements to terminate abruptly at $85 \mathrm{~km}$. The meteor mass was estimated by infrasound measurements to be 526 grams (ReVelle and Whitaker, 1999; Zinn et al, 1999). The zenith angle of the radiant over New Mexico at $3 \mathrm{AM}$ on 17 November was 48 degrees. Then using the expected initial velocity of 70 $\mathrm{km} / \mathrm{s}$ for a Leonid, and substituting these values into Eq. (14), we obtain $A=7.7 \times 10^{-11} \mathrm{~s}^{2} / \mathrm{g}^{2 / 3}$.

It is likely that the bulk density $\rho_{s}$ of a Leonid meteor is $1 \mathrm{~g} / \mathrm{cc}$ or less, and the most likely value of the drag coefficient $C_{D}$ for the particular 526-g case is about 0.6 (see Liepman \& Roshko, 1957). Combining these values with the value of $A$ just determined, we obtain $\frac{f_{Q}}{e}=2.1 \times 10^{-10} \mathrm{~s}^{2} / \mathrm{cm}^{2}$. As noted in the next section, this quantity is the same as the familiar "ablation parameter" $\sigma$.

Now the energy transfer fraction $f_{Q}$ is unknown, but must be smaller than 1 . So the above value of $\frac{f_{Q}}{e}$ implies that the effective ablation energy $e$ must be less than $4.8 \times 10^{9} \mathrm{erg} / \mathrm{g}$. This is about five times smaller than 
the heat of vaporization of ice, and comparable with the heat of fusion. A meteor entering the atmosphere is subject to very large surface shear forces, and the energy required to tear solid particles from the surface may be much less than the heat of vaporization.

\section{COMPARISONS OF VARIABLES WITH PREVIOUS LITERATURE}

In Eq. (3) we chose to define the variable $f_{Q}$ as the fraction of the drag energy that goes into ablation, where $f_{Q}$ $<1$. Ceplecha et al (1998) and earlier authors have chosen instead to define a "heat transfer coefficient" $\Lambda$, along with an "ablation coefficient" $\sigma$, a drag coefficient $\Gamma$, an ablation energy $\xi$, and a "shape-density coefficient" $K$. In terms of our variables

$$
\begin{aligned}
& {[\sigma]_{C}=[\Lambda / 2 \xi \Gamma]_{C}=\left[\frac{f_{Q}}{e}\right]_{Z J R}} \\
& {[\Gamma]_{C}=\left[C_{D} / 2\right]_{Z J R}} \\
& {[\xi]_{C}=[e]_{Z J R}} \\
& {[\mathrm{~K}]_{C}=\left[.604 C_{D} / \rho^{2 / 3}\right]_{Z J R}} \\
& {[\sigma \mathrm{K}]_{C}=[A]_{Z J R}}
\end{aligned}
$$

where Ceplecha et al variables are indicated by subscript $C$, and variables in this paper by subscript $Z J R$.

To integrate their equations, Ceplecha et al assume that $\sigma$ and $K$ are constant. This is equivalent to our assumption that $\frac{f_{Q}}{e}$ and $A$ are constant. The point of departure of our analysis from theirs is that we assume a locally exponential atmosphere (our Eq. (9)), allowing us to proceed further with the integrations.

\section{SOME NUMERICAL EXAMPLES FOR LEONIDS}

Now with this same value of $A$, and choosing a trajectory zenith angle of 48 degrees, for each of a set of meteors with a range of initial masses, we can compute and plot the meteor masses vs. altitude as the ablation proceeds. (The variation of meteor mass with decreasing altitude along the trajectory is given by Eq. (11).) The composite plot is in Figure 1, and it demonstrates, of course, that the larger the initial meteor mass the deeper in the atmosphere it penetrates.

For all six of the cases in Figure 1 the velocities remain essentially constant, decreasing by less than $1 \%$ over the entire time spans until the meteor ablation is complete.

Figure 2 is a composite plot of the energy deposition rates along the trajectories for the same set of meteors. (dU/ds from Eq. 22).

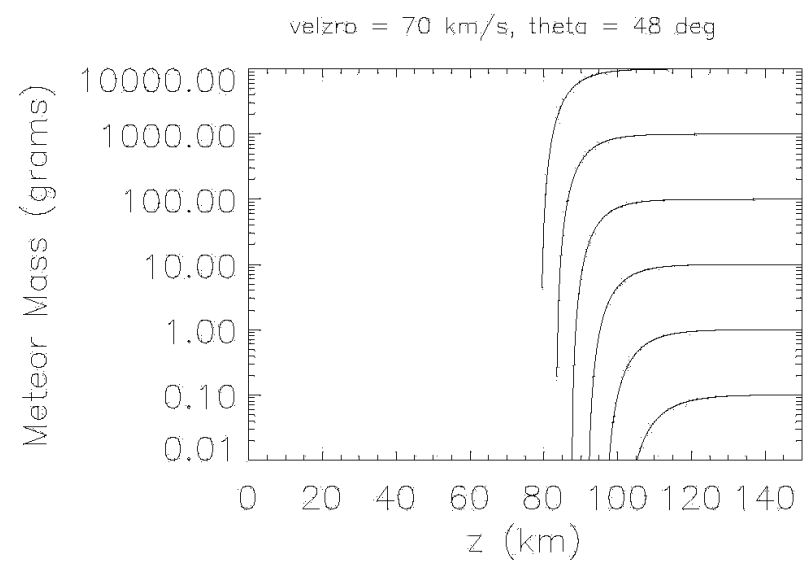

Fig. 1. Plots of instantaneous meteor masses vs altitude for six different Leonid cases with differing initial masses.

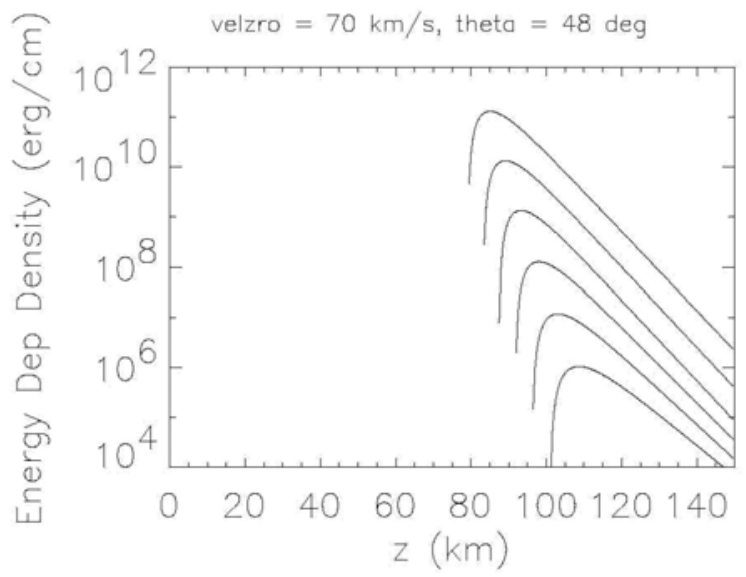

Fig. 2. Plots of energy deposition per unit distance along the trajectories $(\mathrm{erg} / \mathrm{cm})$ for the same six Leonid cases in Fig 1.

This analysis is of course based on the assumption that the meteors do not fragment, and they remain spherical down to the terminal altitude. If a meteor does fragment, then to a first approximation it will behave as an aggregate 
of several smaller meteors, so that their terminal altitudes will not be as deep in the atmosphere as the ones shown in Figure 1. (See discussion in ReVelle, 2001, 2002).

\section{NUMERICAL COMPUTATIONS OF THE CYLINDRICAL EXPANSION OF THE METEOR WAKE}

Having calculated the linear density of deposition of mass and energy along the meteor path, we can investigate the corresponding rate of radial expansion of the wake, and its temperature and luminosity. For this purpose we use a newly-developed cylindrical variant of a radiative transfer and hydrodynamics computer model that we used previously in a spherical version for the study of the 1998 Leonids (Zinn et al, 1999). The numerical algorithm includes 42-frequency group radiation transport coupled with Lagrangian hydrodynamics, using detailed tables of air equation of state and radiative opacity data (as in Zinn, 1973) (meteor materials not included).

For the purposes of the exercise we will consider a large Leonid with a mass of 500 grams, and an initial velocity of $70 \mathrm{~km} / \mathrm{s}$, with a zenith angle of 60 degrees. Then its theoretical terminal altitude should have been about $84 \mathrm{~km}$, and the maximum linear rate of energy deposition should have been at about $90 \mathrm{~km}$. At $90 \mathrm{~km}$ the energy deposition density would have been $5.0 \times 10^{9} \mathrm{erg} / \mathrm{cm}$, and the corresponding mass deposition would have been 2.06 $\mathrm{x} 10^{-4} \mathrm{~g} / \mathrm{cm}$. For the computation it is also necessary to assume an initial mass density for the ablated vapor, which we take to be $0.5 \mathrm{~g} / \mathrm{cm}^{3}$. With these values for input we can run the computation for a set of various altitudes.

One of the outputs of the computation is a set of profiles of temperature vs. radius (assuming cylindrical symmetry) for a specified set of times. Figure 3 is such a set of profiles for the $90 \mathrm{~km}$ cross-section of the meteor wake. The earliest profile, at 10 microseconds, shows the formation of an intense shock wave driven by the rapid expansion of the very hot vapor-air mixture against the lower-density surrounding air. This shock wave subsequently propagates outward, while cooling and radiating strongly at ultraviolet wavelengths (as well as visible and infrared), leading to a radiatively-expanding precursor front ahead of it. At later times, after a few milliseconds, the precursor front develops its own shock wave structure, which continues to propagate outward. One will note that the temperatures at early times in the vicinity of the inner shock wave are in the several- electron-Volt range, although they rapidly cool down to 3000 to 4000 degrees K and lower.

For each of the times represented in the set of temperature profiles there is a corresponding value of distance along the meteor trajectory that the meteor will have moved since passing the $90 \mathrm{~km}$ mark. Therefore, the same set of temperature vs. radius and time results can be replotted in the form of a set of contours of temperature vs. crosswise radial displacement from and distance along the meteor wake. Figure 4 includes three such contour plots showing the temperature distributions behind the meteor when it is at altitudes 86,90 , and $94 \mathrm{~km}$ respectively. One will note that the contours are relatively smooth and spread out along the axial dimension. We take that as a

justification of our approximation of local cylindrical symmetry for the radial expansion. It can also be noted that the wake tends to spread out more rapidly the higher the altitude. 


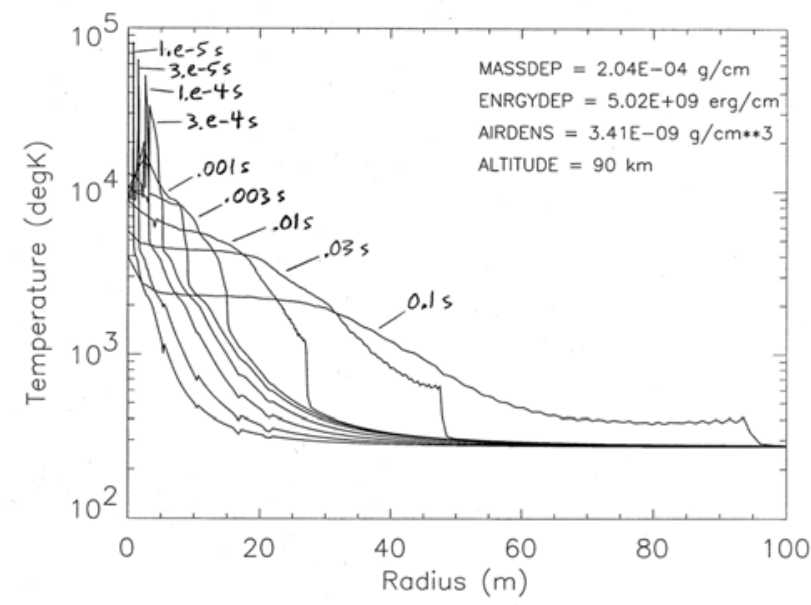

Fig. 3. Profiles of temperature vs radial distance from the meteor path for the 500-g Leonid at eight different times in the $90-\mathrm{km}$-altitude cross section of the meteor trajectory. (The times are 1.e-5, 3.e-5, 1.e-4, 3.e-4, 1.e-3, 3.e-3, .01, .03 , and 0.1 seconds)

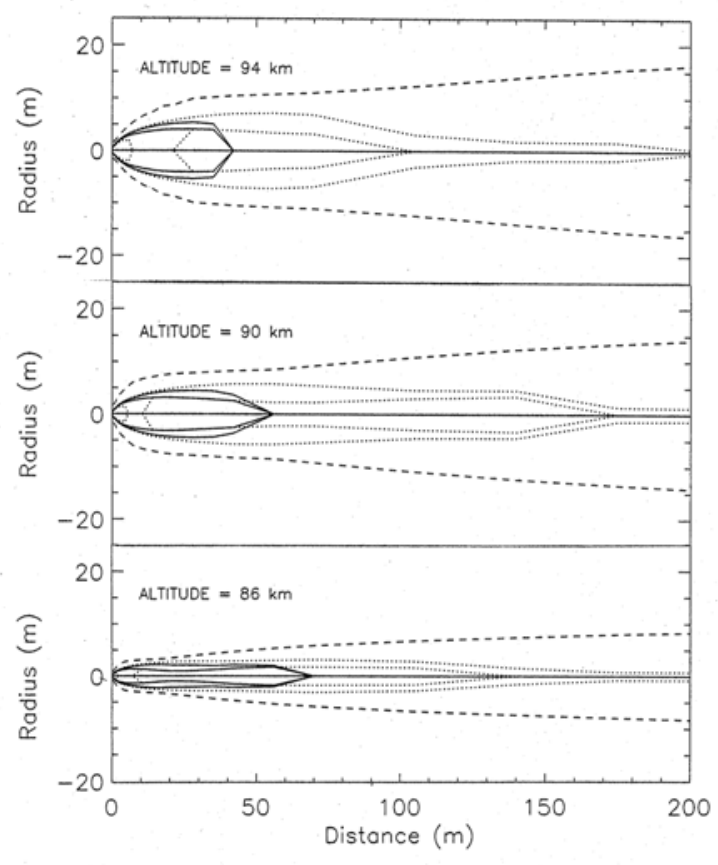

Fig. 4. Plots of contours of temperature for three different snapshots along the meteor trajectory, namely when the meteor is at $86 \mathrm{~km}$, and $90 \mathrm{~km}$, and $94 \mathrm{~km}$, respectively. (20000k contour = solid, $10000 \mathrm{k}$ contour $=$ dotted, $3000 \mathrm{k}$ contour $=$ dashed)

From the same set of rad/hydro computations we can extract values of radiated energy vs. time at visible wavelengths, and also visible radiation efficiencies (i.e. visible light output divided by total energy input) for each of the altitudes. For all the altitudes the computed results show the visible output rate peaking at times before 1 millisecond, and they show a time-integrated visible efficiency of about 13.5 percent, and an infrared efficiency of 40 percent (primarily bremsstrahlen emission). Although at each individual altitude the light output peaks within a millisecond, the light output measured by an observer on the ground will be proportional to the product of the meteor velocity times the total visible emission per unit distance along the trajectory. And the visible emission per unit distance is the visible efficiency times the ablated meteor vapor kinetic energy deposited per unit distance. Thus, from the computed visible efficiencies, together with the values of $d U / d s$ from Eq. (22) (see Figure 2), we can compute the (theoretical) light output vs. time. The computed light output vs. time for the 500-gram Leonid is shown in Figure 5. It shows the light coming out in a pulse with a half-width of about 0.3 second.

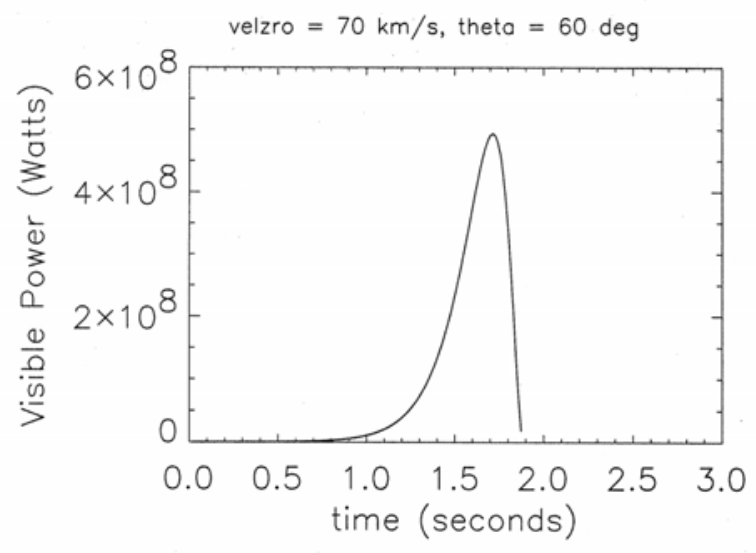

Fig. 5. Computed visible power output vs time for the 500-gram Leonid. 


\section{COMPARISONS WITH DATA, AND DISCUSSION OF DIFFERENCES}

The subject 1998 Leonid was observed at several New Mexico locations with several kinds of equipment. The observations included photometer measurements of light output vs. time, infrasound measurements that were used to infer total energy and mass, radar measurements giving altitude vs. time, and measurements of the long-lasting meteor train. The latter meteor train measurements included high-resolution video and sodium lidar, both from the Air Force Starfire Optical Range (Drummond et al, 2001), and narrow-band sodium line all-sky photographs (Kruschwitz et al, 2001). Some of these data, along with our own preliminary computations, were reported in our 1999 paper.

The inferred mass of 526 grams was based on analysis of the infrasound data, which entails many assumptions of uncertain validity. From the photometer measurements together with the infrasound-derived total energy we extracted a visible efficiency of $1.7 \%$ — considerably smaller than the computed efficiency of $13.5 \%$. The photometer record showed a very smooth visible emission pulse with a half-width of about 0.2 seconds. The smoothness suggests that the meteor did not break up. The video data, of which we were unaware at the time of writing the 1999 paper, showed a trajectory terminal altitude of $85 \mathrm{~km}$.

\section{CHEMISTRY AND LIGHT EMISSION IN THE LATE-TIME METEOR TRAIN}

The computer model also includes time-dependent chemical kinetics, with 687 individual chemical reactions. We compute concentration profiles of 43 different chemical species at selected times, for selected cross-sections along the meteor wake. As an example, Figure 6 shows a set of concentration profiles of several chemical species in the $90 \mathrm{~km}$ altitude cross-section of the train one second after passage of the meteor. The plot shows a highly ionized inner region associated with the expanding meteor vapor + air mixture. Outside of this core region is an extended region with large atomic $N$ and $O$ concentrations produced mainly by photodissociation, and radially-decreasing ion and electron concentrations due to photoionization. There are also elevated concentrations of metastable $N\left({ }^{2} D\right)$ atoms, produced by dissociative recombination of electrons with $N_{2}{ }^{+}$ions, and $N O$ produced by reactions of $N\left({ }^{2} D\right)$ with $\mathrm{O}_{2}$. There are also significant concentrations of metastable $O\left({ }^{1} D\right)$ atoms and $\mathrm{O}_{2}\left({ }^{1} \Sigma\right)$ molecules.

The $N\left({ }^{2} D\right)$ and $O\left({ }^{1} S\right)$ lead to green line emission at 520 and $557.7 \mathrm{~nm}$ respectively, and the $O\left({ }^{1} D\right)$ emits the familiar red line at $630 \mathrm{~nm}$. The $O_{2}\left({ }^{1} \Sigma\right)$ produces band emission in the near infrared (the $O_{2}$ "atmospheric band"). The 2-body reaction of $N O$ with $O$ atoms produces yellow-green continuum emission. From the 1-second concentration profiles in Figure 6 we can estimate the emission intensities which, for the 520, 557.7 and $630 \mathrm{~nm}$ lines are respectively 3.6, 15, and 3.4 kilorayleighs $\left(1\right.$ Rayleigh $=1 \times 10^{6}$ photons $\left./ \mathrm{cm}^{2} \mathrm{~s}\right)$. For the $\mathrm{O}_{2}$ atmospheric bands the estimated intensity is 100 Megarayleighs. For the $\mathrm{NO}_{2}$ yellow-green continuum it is 10 kilorayleighs.

Figure 7 is a similar set of profiles at 66 seconds - also for the $90 \mathrm{~km}$ cross section. From those profiles the estimated intensities are 140 and 13 kilorayleighs respectively for the $557.7 \mathrm{~nm}$ green line and $\mathrm{NO}_{2}$ yellow-green continuum, and the infrared atmospheric band intensity has dropped to 15 kilorayleighs. The $520 \mathrm{~nm}$ and $630 \mathrm{~nm}$ intensities have dropped below one kilorayleigh.

A prominent feature in Leonid meteor train spectra is the $589.3 \mathrm{~nm}$ doublet of atomic sodium. The chemistry model represented in Figures 6 and 7 did not include any sodium species. However, more recently we have extended it to include $\mathrm{Na}, \mathrm{Na}+\mathrm{NaO}, \mathrm{NaO}_{2}$, and $\mathrm{NaOH}$. Preliminary runs with the extended model are in progress, but it is already apparent that to model the sodium airglow it will also be necessary to include turbulent diffusion,

which so far we do not include. Another model that includes sodium species was described by Kruschwitz et al (2001). 


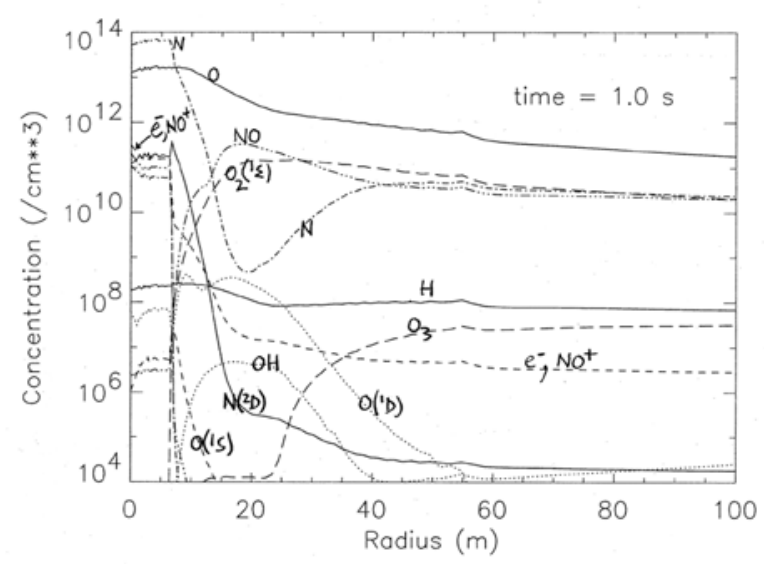

Fig. 6. Computed radial profiles of concentrations of several chemical species at 1 second after passage of the meteor at $90 \mathrm{~km}$ altitude.

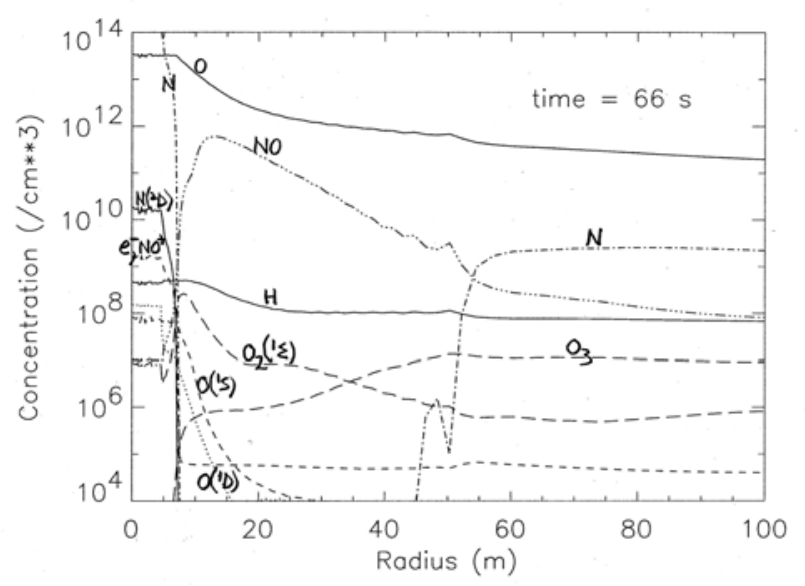

Fig. 7. Computed radial profiles of concentrations of several chemical species at 66 seconds after passage of the meteor at $90 \mathrm{~km}$ altitude.

\section{DISCUSSION OF APPROXIMATIONS}

The present model includes several more-or-less strenuous assumptions. One is our use of the equations of hydrodynamics in a parameter regime where molecular collision means free paths are comparable with, and in many cases larger than the dimensions of the meteors. The collision mean free path for air molecules is about one centimeter at an altitude of $82 \mathrm{~km}$. The 526-gram meteor (if spherical and with a density of $1 \mathrm{~g} / \mathrm{cm}^{3}$ ) has a radius of $5 \mathrm{~cm}$, so even at its terminal altitude it would appear to be only barely into the hydrodynamic fluid regime, assuming that the relevant collision cross sections were those of neutral molecules with each other. On the other hand, for a Leonid entering the atmosphere the bow shock stagnation temperature is about $8 \mathrm{eV}$, so the air behind the shock is almost completely ionized. That being the case the most important and frequent collisions are those of electrons with neutral molecules (as well as with the ions behind the shock). The electrons are coupled to one another through the geomagnetic field, which is partially picked up and swept along by the plasma, and they are coupled to the ions through electrostatic forces. By virtue of these processes the meteor-air interaction should be effectively collisional at an appreciably higher altitude than that given by the simple unit-Knudsen-number criterion.

Our calculations of the meteor ablation rate and the derivation of the parameter $A$ are based on the assumption that the meteor does not break up, and that the ablated material consists of vapor or small particles. This assumption is questionable.

For the numerical computations of the meteor wake expansion it was necessary to assume a value for the initial density of the meteor vapor-air mixture, which we took to be $0.5 \mathrm{~g} / \mathrm{cc}$. Since the numerical scheme conserves mass and energy, the results should not be very sensitive to this assumption. However, we have not done a sensitivity study with respect to variations of this initial density. A related question concerns the possible role of Taylor and shear instabilities, which would be expected to occur, and which we do not include.

Regarding the early-time radiation emission, we noted previously that the dominant emission is associated with bremsstrahlen from the intensely hot and fully ionized inner region of the wake. But we are aware of the fact that the measured spectra of Leonids are dominated by metallic line emissions (Borovicka and Jenniskens, 2000; Abe et $a l, 2000)$. To include the line emissions in detail is beyond the capabilities of the radiation transport model, but the computed results do suggest that most of the emitted energy is in bremsstrahlen.

\section{EXTENSION OF THE MODEL TO SPORADIC METEORS}

Since the present model seems to work fairly well for Leonids, it is reasonable to ask if it can be extended to other meteor classes. In general, these will differ from Leonids mainly with respect to their velocity, mass density, and energy of ablation. If, for a typical stony meteor, we take $v_{\infty}=20 \mathrm{~km} / \mathrm{s}, \rho_{s}=3 \mathrm{~g} / \mathrm{cm}^{3}, e=8 \times 10^{10} \mathrm{erg} / \mathrm{g}$, and $\sigma=$ $5 \times 10^{-12} \mathrm{~s}^{2} / \mathrm{cm}^{2}$ (from Ceplecha et al, 1998) then we find that relative to Leonids, these meteors penetrate more deeply into the atmosphere before suffering significant ablation. However, because of their smaller value of $\sigma$, according to Eq. (5) the velocity begins to drop below $v_{\infty}$ when only a modest amount of mass has been lost by ablation. When this point is reached it is no longer valid to assume that $v$ is constant, so that Eqs. (7) through (10) 
no longer apply. Then to continue the calculation one needs to resort to numerical integration of Eqs. (1) and (3). We have written a simple computer code to do that, and used the code to compute $m$ vs. $z$ and $v$ vs. $z$ for an array of meteoroids with the same $v_{\infty}, \theta, \rho_{s}$, and $\sigma$, but an assortment of different initial masses. Some results are plotted in Figures 8 and 9. They show that these meteors tend to penetrate to much lower altitudes than do Leonids with the same values of initial mass. They also show the velocities hold relatively constant until $\mathrm{m} / \mathrm{m}_{\infty}$ has dropped to about 0.1 , and thereafter they decrease rapidly. But ablation essentially ceases when the velocity drops below $10 \mathrm{~km} / \mathrm{s}$, although by that time $m / m_{\infty}$ has been reduced to about $1 \times 10^{-5}$. After the ablation ceases and $m$ and $S$ are constant the velocities continue to drop until they reach the terminal velocity given by

$$
v_{\text {termnl }}=\sqrt{\frac{2 m g \cos \theta}{C_{D} \rho S}}
$$

The mass vs. altitude plots show, that in each case, only a fraction of about $10^{-5}$ of the original mass reaches the ground. Thus for a mass of $1 \mathrm{~kg}$ to reach the ground the original mass would have had to be 100 metric tons, and the total energy deposited would be 5 kilotons.

These calculations have assumed that the meteor does not break up. However, we have also computed the dynamic pressure $\frac{1}{2} \rho v^{2}$ vs. altitude for each of these meteors - on their leading surfaces. For the 100 metric ton case we find that the front-to-rear pressure differential reaches about 500 atmospheres, which is probably more than sufficient to cause fragmentation.

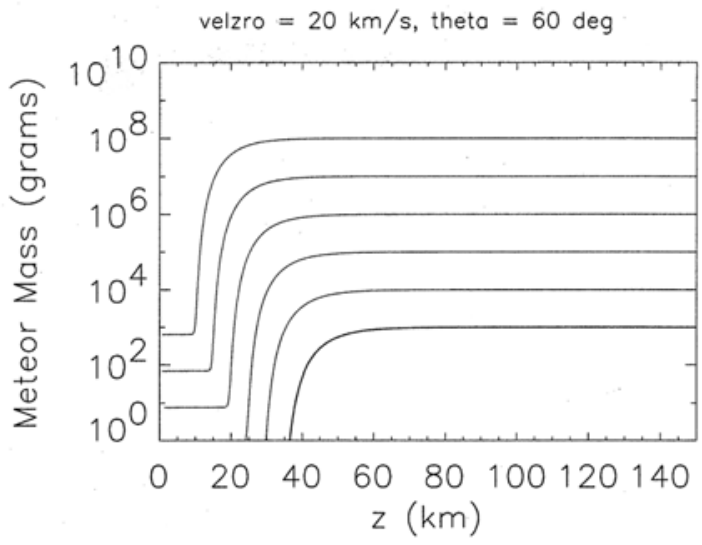

Fig. 8. Computed instantaneous masses vs altitude for six different sporadic meteor cases with differing initial masses.

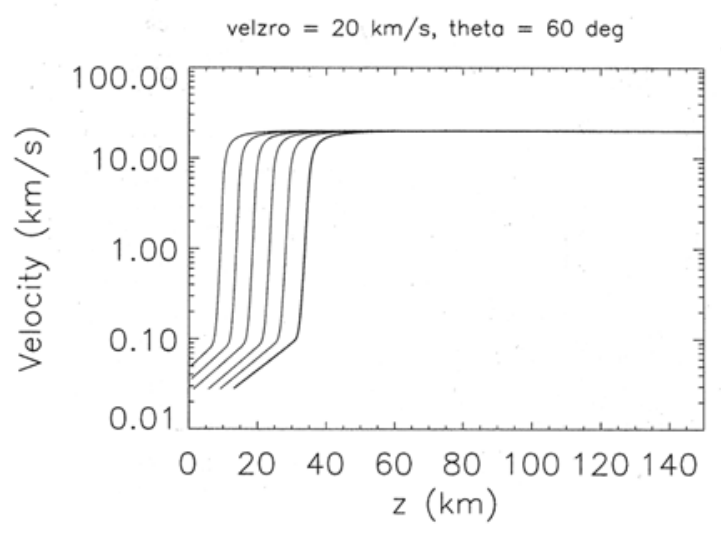

Fig. 9. Computed instantaneous velocities vs altitude for the same six sporadic meteor cases as in Fig. 8.

\section{CONCLUSIONS}

We have shown that the predominant process of energy deposition in the atmosphere by a Leonid meteor is the conversion of the kinetic energy of the ablated particles and/or vapor into internal energy as they are stopped by the background air. The energy required to completely vaporize the meteor is negligible compared to its initial kinetic energy. The stopping of the ablated vapor is almost instantaneous, and results in very high temperatures, which give rise to a radially (cylindrically) expanding shock wave. We have described numerical computations of the radiative-hydrodynamic evolution of this structure, as well as its chemical evolution, and presented temperature contours in the wake behind the meteor at three different altitudes along its trajectory. The computed results also include a value of the visible radiation output fraction (13.5\%), which is eight times larger than a recent empirical estimate. We have also derived equations to define an ablation parameter $A$, which should have a common value for all meteors of a given family. Given a set of measured values of meteor velocity $v_{\infty}$ trajectory zenith angle $\theta$, mass $m_{\infty}$ (derived from infrasound data), and terminal altitude $z_{T}$, we determined the fitted value of $A$, which should be applicable for all Leonids. 


\section{REFERENCES}

Abe, S., H. Yano, N. Ebizuka, and J. Watanabe, "First results of high-definition TV spectroscopic observations of the 1999 Leonid meteor shower", Leonid Storm Research, (Jenniskens, Rietmeijer, Brosch, and Fonda - eds), Kluwer Academic Publishers, 2000.

Borovicka, J. and P. Jenniskens, "Time-resolved spectroscopy of the Leonid fireball afterglow", Leonid Storm Research (Jenniskens, Rietmeijer, Brosch and Fonda - eds), Kluwer Academic Publishers, 2000.

Bronshten, V. A., Physics of Meteoric Phenomena, D. Reidel Pub. Co., 1983.

Ceplecha, Z., J. Borovicka, W. G. Elford, D. O. ReVelle, R. L. Hawkes, V. Porubcan, and M. Simek, "Meteor Phenomena and Bodies", Space Science Reviews 84, 327-471, 1998.

Drummond, J., B.W. Grime, C.S. Gardner, A. Z. Liu, X. Chu, M. C. Kelley, C. Kruschwitz, and T. J. Kane, "Observations of persistent Leonid meteor trails III: The 'Glowworm'”, J Geophys. Res. in press 2002.

Kruschwitz, C. A., M. C. Kelley, C. S. Gardner, G. Swenson, A.Z. Liu, X. Chu, J. D. Drummond, B. W. Grime, W. T. Armstrong, J. M. C. Plane, and P. Jenniskens, "Observations of persistent Leonid meteor trails II: Photometry and numerical modeling”, J. Geophys. Res., 106, 21525-21541, 2001.

Landau, L. D. and E. M. Lifshitz, Fluid Mechanics, Pergamon Press, 1959 (p169).

Liepman, H. W. and A. Roshko, Elements of Gas Dynamics, John Wiley \& Sons, New York, 1957.

ReVelle, D. O., "Theoretical Leonid entry modeling”, in Proceedings of the Meteoroids 2001 Conference, Kiruna, Sweden, Aug. 2001 (B. Warmbein, ed), pp 149-154 and 179-184.

ReVelle, D. O., "Fireball dynamics, energetics, ablation, luminosity and fragmentation modeling", in Proceeding of the ACM Conference, Berlin, Germany, Aug. 2002 (B. Warmbein, ed), in press (2002)

ReVelle, D. O., and R. W. Whitaker, "Infrasonic detection of a Leonid bolide: 1998 November 17" Meteoritics and Planetary Science 34, 995-1005, 1999.

Spurny, P., H. Betlem, J. V. Leven, and P. Jenniskens, "Atmospheric behavior and extreme beginning heights of the thirteen brightest photographic Leonid meteors from the ground-based expedition to China", Meteoritics and Planetary Science 35, 243-449, 2000

Zinn, J., J. Wren, R. Whitaker, J. Szymanski, D. O. ReVelle, W. Priedhorsky, J. Hills, G. Gisler, S. Fletcher, D. Casperson, J. Bloch, R. Balsano, W. T. Armstrong, C. Akerlof, R. Kehoe, T. McKay, B. Lee, M. C. Kelley, R. E. Spalding, and S. Marshall, "Coordinated observations of two large Leonid meteor fireballs over northern New Mexico, and computer model comparisons", Meteoritics and Planetary Science 34, 1007-1015, 1999.

Zinn, J., "A finite difference scheme for time-dependent spherical radiation hydrodynamics problems" $J$. Computational Phys. 13, 569-590, 1973.

Correspondence concerning this article should be sent to jzinn@lanl.gov. 